\title{
Du prix de Rome au quai de la Piperie, Mathurin Crucy, entrepreneur de construction navale à Nantes
}

The Prix de Rome on the Quai de la Piperie, Mathurin Crucy, an Entrepreneur of naval Construction in Nantes

\section{David Plouviez}

\section{(2) OpenEdition \\ Journals}

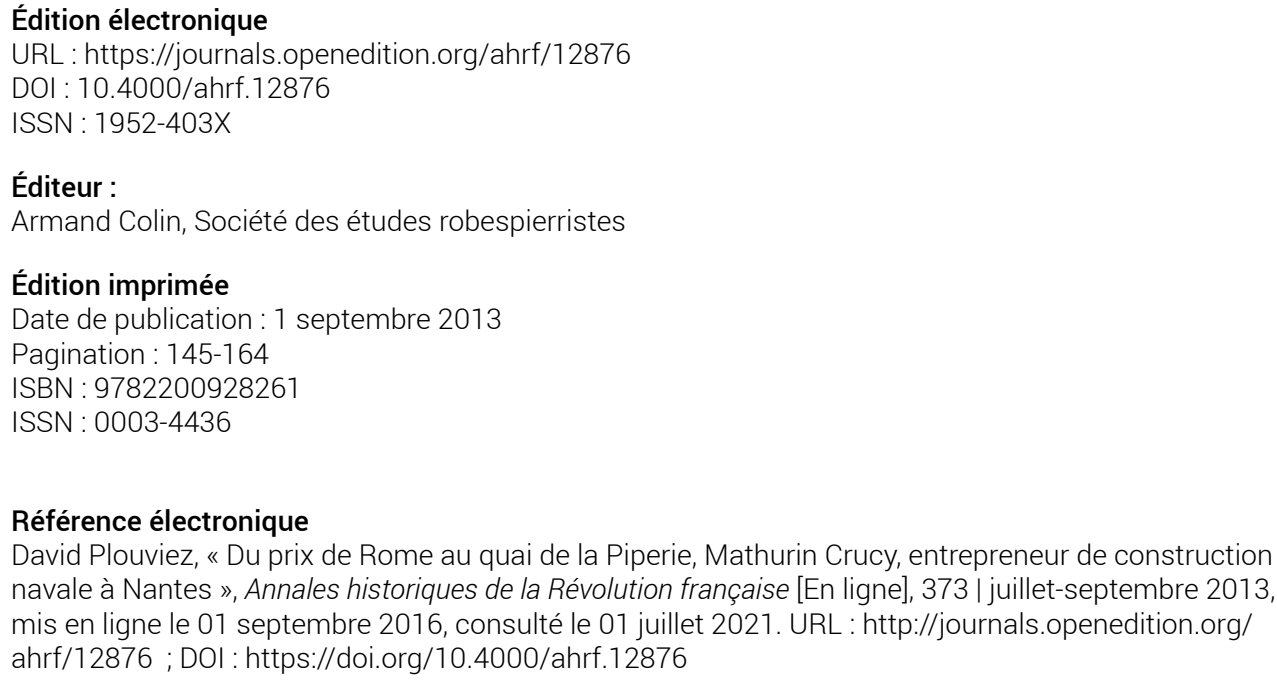

Référence électronique mis en ligne le 01 septembre 2016, consulté le 01 juillet 2021. URL : http://journals.openedition.org/ ahrf/12876 ; DOI : https://doi.org/10.4000/ahrf.12876 


\title{
DU PRIX DE ROME AU QUAI DE LA PIPERIE, MATHURIN CRUCY, ENTREPRENEUR DE CONSTRUCTION NAVALE À NANTES
}

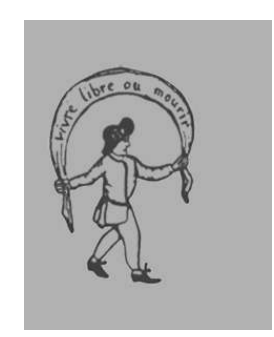

David PLOUVIEZ

\begin{abstract}
Établi à Nantes à partir de 1780 et partie prenante de l'entreprise familiale de construction navale au service de l'État, Mathurin Crucy livre un récit autobiographique intitulé L'Exorde dans lequel il détaille ses années de formation, son activité d'architecte-voyer et sa participation dans la société L. Crucy frères jusqu'à la faillite de cette dernière et la mésentente qui en découle avec sa famille à la fin de l'Empire. Ce document de for privé se révèle indispensable pour pénétrer l'intimité d'un des acteurs au cœur de l'effort de guerre naval de la Révolution et de l'Empire. En marge de cette charge contre ses frères et des différents gouvernements qui se sont succédés à partir de 1793, l'Exorde offre la possibilité d'approcher d'une manière originale le fonctionnement des chantiers navals privés à vocation militaire.
\end{abstract}

Mots-clés : Mathurin Crucy, for privé, architecte, construction navale, Nantes, Marine

Architecte-voyer de Nantes et partie prenante d'une entreprise de construction navale au service de la Marine, Mathurin Crucy fut une figure emblématique locale autant qu'il a été un rouage essentiel dans la capacité de la France à projeter vaisseaux et frégates sur les théâtres militaires pendant la Révolution et l'Empire. Les archives de cette aventure industrielle et commerciale constituent un fond entrepreneurial disparate mais incontournable pour en saisir la vie et les péripéties des années 1780 à 1814. Partageant les responsabilités, les gains comme les pertes, Mathurin et ses deux frères, Louis et Antoine, furent à la tête des premiers chantiers 
de sous-traitance de l'époque autant par le nombre d'unités lancées que par leur tonnage ${ }^{1}$. Dans la masse documentaire laissée par la société L. Crucy Frères, une partie des papiers privés de Mathurin a été classée dans une section spécifique ${ }^{2}$. Outre les archives relevant de son premier métier (correspondance, plans, dessins...) on trouve un document manuscrit intitulé L'Exorde où il évoque son parcours depuis sa formation jusqu'à sa participation dans l'entreprise de construction navale. Matériellement, il s'agit d'un cahier d'une dizaine de pages brouillonnes relevant du genre autobiographique où l'auteur s'embarrasse peu de la chronologie et de la précision des événements décrits ${ }^{3}$. On peut s'interroger sur les conditions de rédaction d'un tel document dans lequel Mathurin se présente, en 1813, comme la victime des différents gouvernements depuis 1793 et de ses deux frères qui l'ont privé de son métier d'architecte ainsi que d'une partie de sa fortune. Bien décidée à solder ses affaires sur fond de mésentente générale, la fratrie s'engage dans un règlement judiciaire et il s'agit, pour Mathurin, de fournir des arguments à ses avocats. La rédaction de ce document s'inscrit sans doute dans ce contexte, même s'il ne semble pas avoir été utilisé. En parallèle du caractère informatif essentiel pour rétablir l'historicité du parcours de Mathurin et de l'entreprise Crucy, l'Exorde offre un deuxième niveau de lecture relatif à la conscience que Mathurin s'est porté à lui même. Rétrospectivement, le scripteur revient tout au long de son texte sur la construction de son identité d'architecte et évoque en creux la violence qu'il a subie pour pouvoir exercer son métier sereinement. Que celle-ci soit surévaluée ou objective, il reste qu'elle se décline au travers des relations familiales, des rapports entretenus par Mathurin avec les gouvernements révolutionnaires, le Consulat ou l'Empire ainsi que dans la relation complexe nouée avec ces derniers dans le cadre des commandes de navires militaires.

(1) Plusieurs décomptes des unités lancées par les Crucy ont été réalisés en 1808 et 1812 qui omettent de nombreuses constructions secondaires. Pour le reste, on dénombre 9 vaisseaux de 74 canons complets, 4 membrures de vaisseaux et 28 frégates parmi les navires les plus lourds. À ces chiffres il faut ajouter de nombreuses flûtes, corvettes, bricks, goélettes, AD Loire-Atlantique $121 \mathrm{~J} 21$, fonds Crucy, chantiers navals et négoce.

(2) AD Loire-Atlantique 121 J 4-20, fonds Crucy, Mathurin Crucy, architecte (1749-1826).

(3) Pour aborder ce genre, on se reportera à deux publications récentes : Michel CASSAN (dir.), Écritures de familles, écriture de soi (France-Italie, XVI ${ }^{e}-X I X^{e}$ siècles), Limoges, PULIM, 2011 et Sylvie MouYSSET, Jean-Pierre BARDET, François-Joseph RUGGIU (dir.), Car c'est moy que je peins. Écriture de soi, individu et liens sociaux (Europe, $X V^{e}-X X^{e}$ siècle), Toulouse, Méridiennes, 2010. 


\section{Des années de formation à celles de l'entreprise de construction navale : perception de soi et construction identitaire}

\section{Les belles années de l'apprentissage à Paris et à Rome}

Si le ton d'ensemble de l'Exorde est empreint de pessimisme, il reste que les lignes que Mathurin consacre à ses années de formation révèlent une période d'allégresse et de travail passionné. Il n'est pas impossible que l'exaltation de ces moments lui permette a posteriori de justifier son renoncement et d'indiquer la mesure de son sacrifice quand il dut abandonner sa charge d'architecte-voyer en 1800 pour se consacrer pleinement à la co-direction des affaires familiales. Au delà, l'écriture de l'Exorde témoigne d'une construction identitaire inattendue de la part de son auteur. Celui-ci ne se définit pas en fonction d'une ascendance familiale ou d'une appartenance sociale mais par son métier d'architecte. Cette perception sincère de lui-même est très nette tout au long de cette autobiographie se plaçant naturellement au centre de son propos mais en donnant le sentiment d'une évidence quant à son destin. Deuxième de la famille Crucy, Mathurin se consacre à des études d'architecture, vraisemblablement à la suite d'un cours de dessin dispensé à Nantes par la Société d'Agriculture, du Commerce et des Arts ${ }^{4}$. En 1767, il se rend à Paris pour poursuivre sa formation auprès de Jean-Jacques Bachelier qui dirige alors l'école gratuite de dessin ouverte l'année précédente ${ }^{5}$. L'entrée à l'Académie royale d'architecture date probablement de 1771 mais cette période est peu documentée. On y retrouve l'élève Mathurin en 1772, se présentant en 1773 au prix de l'Académie où il termine deuxième, avant d'être lauréat en 1774, son billet pour l'Académie de France à Rome. Sur cet épisode, Mathurin omet d'évoquer la puissance des réseaux de son père, pourtant déterminante pour la construction de son parcours. Sans nier le talent du jeune homme, le choix de l'architecture est loin de relever d'un heureux hasard. Depuis son arrivée à Nantes, Jean Crucy père est lié à Jean-Baptiste Ceineray qui devient architecte de la ville en 1759. Cette amitié se concrétise par de nombreuses affaires - notamment le commerce du bois - mais également par la formation du jeune Mathurin au métier de Ceineray. La succession de ce dernier était convoitée par les Crucy.

(4) Claude Cosneau, Mathurin Crucy, 1749-1826, architecte nantais néo-classique, Musées départementaux de Loire-Atlantique/Musée Dobrée, Nantes, 1986, p. 45. Hayot, 2004.

(5) Ulrich LEBEN, L'École royale gratuite de dessin de Paris, Saint-Rémy-en-l'eau, Monelle 
Une fois ses compétences démontrées, le jeune homme entre à l'Académie grâce à son mentor et au concours de Joseph-Marie Vien, directeur de l'établissement à Rome à partir de 1775.

Mathurin fait de ces années une période fondatrice d'autant plus qu'elle est couronnée de succès. L'architecture apparaît comme un puissant facteur identitaire et il n'est pas excessif d'affirmer qu'il s'agit de sa raison d'être. Dès lors, la répartition des rôles décidée dans la famille en 1774 était naturelle : «Ma sœur Peccot avait trois garçons, le père pouvait mourir, nous avions donc arrêté que je serais architecte, que mon frère Jean serait à la tête des affaires de la maison, Louis peintre et Antoine sculpteur $»^{6}$. Mathurin pouvait poursuivre serein son apprentissage à Rome entre 1775 et 1779 avant de revenir prendre sa place à Nantes aux côtés de Ceineray.

\section{Travailler pour la Marine : une remise en cause de son identité}

Dans ce contexte, tout événement empêchant Mathurin d'exercer son art est vécu comme une violence insupportable. La mort de Jean-Gilbert en 1783, le premier fils Crucy, vient remettre en cause l'organisation familiale. Mathurin devient l'aîné de la fratrie ; il est tout naturellement désigné pour prendre la direction de l'entreprise. Or, depuis 1780, il est architectevoyer, un poste convoité depuis si longtemps qu'il lui est impossible de l'abandonner. Il troque donc sa place au profit de Louis : "Cette mort [celle de Jean-Gilbert] changea tout le plan que je m'étais formé. Il fallut rappeler Louis à qui je laissais l'option lui ou moi. Mon père était déjà vieux, il ne pourrait suffire à ce travail, Louis vint donc se mettre à la tête de la maison $»^{7}$. Mais finalement, Mathurin se soustrait à ses responsabilités pour peu de temps. Héritant d'une entreprise prospère, Louis et Antoine décident de lui donner davantage d'envergure. Le déclenchement de la Révolution et l'annonce imminente de la déclaration de guerre contre l'Angleterre offrent une occasion que les Crucy ne manquent pas. Le gouvernement de la Convention, afin de soutenir l'effort de construction dans les arsenaux, décide de sous-traiter des unités légères dans les ports secondaires. Poursuivant son activité d'architecte au service de Nantes, Mathurin semble aborder cette nouvelle perspective professionnelle avec enthousiasme comme le montre le passage de l'Exorde relatif à cet épisode : «Le gouvernement ayant le dessein de faire construire dans les ports secondaires des frégates et des corvettes, nous fîmes notre soumission,

(6) AD Loire-Atlantique $121 \mathrm{~J}$ 22-23 folio 1, fonds Crucy, Exorde (1813).

(7) Ibidem. 
notre marché passé nous formâmes des établissements à la Basse-Indre. Il fallut établir une nouvelle cale, faire des magasins, une salle de gabarit, et en peu de temps, ce chantier fut en pleine activité $\gg^{8}$. S'esquisse ici une vision totalisante, voire totalitaire, de l'architecture. Mathurin a cru pouvoir mettre ses connaissances au service de cette société, notamment pour la conception des navires, estimant que les principes de construction d'un immeuble ne différaient pas de ceux d'un vaisseau ou d'une frégate ${ }^{9}$. Par ailleurs, en exerçant à Nantes, il est familier des infrastructures portuaires, en témoigne son premier projet pour la ville consistant en un aménagement des quais du bourg de Chantenay pour accueillir des chantiers et ainsi désengorger ceux de la Chézine ${ }^{10}$. Sa déception est à la hauteur de son enthousiasme. Mathurin ne conçoit pas de navires et devient en réalité l'un des gestionnaires de l'entreprise à laquelle il est contraint de consacrer de plus en plus de temps jusqu'à céder sa charge d'architecte-voyer en 1800 au profit de son neveu, au grand regret des autorités municipales ${ }^{11}$. De son propre aveu, ses voyages à Paris pour signer de nouveaux marchés et collecter de l'argent frais se multiplient et peuvent durer entre « six et sept mois $\gg^{12}$. Loin de Nantes, il lui est de plus en plus difficile de s'investir pleinement dans l'embellissement de la ville, ce qui ne l'empêche pas d'avoir un bilan plus qu'honorable ${ }^{13}$. De fait, ses connaissances en architecture urbaine sont inutiles à la bonne marche des chantiers navals, ceux-ci se contentant d'exécuter des plans tracés par les ingénieurs de la Marine. Cette dépossession a été vécue difficilement par Mathurin et a fondé sa rancœur à l'égard du ministère de la Marine et des différents

(8) Ibid., folio 2.

(9) Ibid., À l'occasion de son exil en 1793, Mathurin semble occuper son temps à la nouvelle activité de la famille : « [...] Je restai huit jours à finir mon travail déjà commencé et vins reprendre mes fonctions, mais avec la ferme résolution de partager mon temps à l'étude de l'architecture navale, qui a beaucoup de rapport avec l'architecture civile et dans l'espace de deux ans, j'avais dessiné depuis un canot jusqu'à un vaisseau de 120 canons. ».

(10) AM Nantes, série II/165 pièce $\mathrm{n}^{\circ} 19$, Plan de projet pour la construction, les quais et rues depuis vis à vis le bourg de Chantenai jusqu'aux nouvelles Salorges, par Crucy (21 octobre 1780).

(11) « Nous sommes sincèrement affligés mes adjoints et moi de ce que les engagements que vous avez contractés avec le gouvernement vous obligent de renoncer à une place que vous occupiez avec tant de distinction [...] Nous sentons, citoyen, tout le prix de l'offre obligeante que vous nous faites d'aider votre successeur de vos lumières et de vos conseils et nous l'acceptons avec reconnaissance », BM Nantes, Manuscrit 2204 pièce $n^{\circ} 51$, le maire de Nantes Fillonneau à Mathurin Crucy (30 août 1800).

(12) AD Loire-Atlantique $121 \mathrm{~J} 22-23$, fonds Crucy, Exorde (1813).

(13) Parmi les nombreuses réalisations de Mathurin Crucy, il faut mentionner la rénovation du quartier Graslin et la construction de son théâtre néo-classique, le cours Cambronne, la Bourse, la halle aux blés, la poissonnerie... Pour une vision exhaustive : Claude CosNEAU, op. cit. 
gouvernements qui se succèdent de 1793 à l'Empire, une antienne qui traverse l'Exorde.

\section{Le projet Paimboeuf-Saint Nazaire, une résurrection?}

Travailler pour l'État dépossède Mathurin de ce qu'il sait faire de mieux et pour lequel il s'est préparé depuis longtemps. Les passages de l'Exorde soulignant cette relégation sont à la hauteur de la blessure ressentie. Toutefois, le ministre de la Marine Decrès semble offrir à Mathurin une parenthèse dans ce long désenchantement en lui commandant un rapport sur la possibilité d'installer des cales de construction dans les environs de Paimboeuf et de Saint-Nazaire. Le paragraphe consacré à cette mission dans l'Exorde est d'importance même si le flou règne une nouvelle fois quant à la date précise. Cette reconnaissance topographique et nautique est l'élément que Mathurin utilise dans son autobiographie pour justifier de son ressentiment envers son frère, Louis, dont le fils aurait utilisé les mémoires techniques rédigés par son oncle pour fonder une entreprise de construction concurrente à Paimboeuf fin 1805. Or le lien entre ce projet industriel et le travail de Mathurin est loin d'être évident dans la mesure où les plans qu'il trace sont postérieurs. D'autres sources indirectes permettent d'envisager la reconnaissance de l'estuaire au début 1808, Mathurin ayant défendu ses conclusions auprès de Napoléon qui fit une descente de la Loire jusqu'à Paimboeuf à l'occasion de son voyage à Nantes les 9 et 10 août de la même année. Au reste, la volonté d'installer un chantier de construction au débouché de la Loire n'était pas une affaire neuve. L'ensablement du fleuve rendait périlleux le lancement d'unités lourdes à Nantes, Basse-Indre, Indret et même Couëron ${ }^{14}$. Sous Napoléon deux rapports que Mathurin connaissait avant l'exécution de sa propre mission de reconnaissance, aboutissent à des conclusions contradictoires. Un premier travail dirigé par le contre-amiral Missiessy et le capitaine de vaisseaux La Bretonnière concluait à l'impossibilité d'établir des chantiers capables de lancer des vaisseaux de 74 canons dans l'estuaire ; les deux ingénieurs des Ponts et Chaussées Mathurin Groleau et Jean-Sébastien Goury concluaient différemment en $1806^{15}$. La mission de Crucy devaitelle servir à départager les deux précédentes enquêtes ? Faute de saisir clairement les motivations du gouvernement, il est difficile de conclure quoi

(14) Anne VAUTHIER-VÉZIER, L'estuaire et le port. L'identité maritime de Nantes au XIX siècle, Rennes, PUR, 2007, p. 39-79.

(15) AM Nantes série 03/carton 18, cité par Yves Cossé, op. cit., p. 189. 
que ce soit sur cette nouvelle commande. À ce stade de l'écriture, l'Exorde change considérablement de forme ${ }^{16}$, évoquant avec une exceptionnelle précision la méthodologie déployée par l'architecte. Au moment de la rédaction de son autobiographie, Mathurin sait que son travail a été soumis à l'ingénieur des Ponts et Chaussées Gaspard de Prony qui reconnut son application, abonda dans son sens pour bien des idées d'aménagement mais resta réservé pour d'autres. Compte tenu de ce que représente l'architecture pour Mathurin, il est évident qu'il tente de démontrer sa probité a posteriori.

Le rapport envoyé, Mathurin ne recevra pas de réponse. La qualité de son travail n'est sans doute pas à remettre en question. En 1808, l'Empire peine à engager de nouveaux grands travaux. Malgré l'intérêt manifeste de Napoléon pour le lancement d'un grand port à Saint-Nazaire ${ }^{17}$, celui-ci ne se développera pas avant $1845^{18}$. Par ailleurs, Mathurin a sans doute été victime des forces économiques nantaises bien décidées à éviter le développement portuaire de l'embouchure de la Loire, synonyme, à terme, de déclassement de Nantes dans sa double fonction d'entrepôt et de port re-distributeur. Sous le feu croisé de la Chambre et du Tribunal du commerce, Mathurin est accusé de favoriser des intérêts particuliers. La première attaque vient de la Chambre de commerce en juillet 1808 qui expose tous les inconvénients du projet à l'Empereur ${ }^{19}$. Début septembre 1808, le rapport de Jacques François intitulé Réflexion sur le projet d'établir à Paimboeuf des chantiers de construction, des bassins, un dépôt d'approvisionnement et de munitions navales $^{20}$ assène un dernier coup à l'idée de développer un grand port dans l'estuaire. Entre son impossibilité de participer à l'élaboration des plans des navires et l'absence de résonance de son rapport sur Paimboeuf et Saint-Nazaire, Mathurin ressort meurtri de sa relation avec la Marine. Ces passages de l'Exorde contribuent à construire l'image d'un homme victime de la violence ordinaire de l'administration, sourde au talent de l'architecte. Le procédé d'écriture est récurrent : Mathurin développe ses ambitions en montrant son implication et sa bonne volonté pour terminer en évoquant

(16) AD Loire-Atlantique $121 \mathrm{~J} 22-23$ folios 4-5, fonds Crucy, Exorde (1813).

(17) L'historien Camille Mellinet rapporte l'entrevue de Mathurin Crucy avec Napoléon où ce dernier aurait affirmé à propos de Saint-Nazaire que «si un projet m'eût été communiqué il y a quelques années, j'aurais maintenant sept vaisseaux de plus. », cf Camille MELLINET, Histoire de la commune et de la milice de Nantes, Nantes, Camille Mellinet éd., tome 12, p. 103-104 (sans date, les douze volumes ont été publiés entre 1839 et 1843).

(18) Guy SAUPIN, « Naissance d'une ville-port atlantique : Saint-Nazaire, avant port et entrepôt (1845-1883) », dans Martine ACERRA, Guy SAUPIN, Guy MARTINIÈRE, Laurent VIDAL, Les villes et le monde du Moyen Âge au XX $X^{e}$ siècle, Rennes, PUR, 2011, p. 189-209.

(19) AD Loire-Atlantique, $106 \mathrm{~J} 74$ (juillet 1808).

(20) Cité par Yves CossÉ, op. cit., p. 191. 
l'absence de considération de ses interlocuteurs. Le rapport à l'égard du ministère de la Marine est fort éclairant. L'image communément véhiculée des relations entre la Marine et ses partenaires économiques depuis la fin du $\mathrm{XVII}^{\mathrm{e}}$ siècle est le plus souvent négative, nourrie par les défauts de paiement ou des exigences qualitatives considérées comme démesurées par les fournisseurs. Or, la traduction littérale de ces tensions n'apparaissait qu'au détour des échanges entre les administrateurs de la Marine ou à l'occasion des litiges. Sans présumer d'un dépouillement complet de la base de données consacrée aux écrits du for privé ${ }^{21}$, il apparaît que l'Exorde est l'un des premiers textes à évoquer de façon aussi détaillée ces rapports difficiles.

\section{L'expérience politique de la Révolution}

\section{Mathurin et la Révolution française : vivre un événement politique par le prisme de son art}

Mathurin Crucy ne porte aucun jugement politique sur la période qu'il traverse et son avis voire ses états d'âme ne transparaissent qu'à l'aune des difficultés qu'il a lui même rencontrées, pendant la Terreur notamment. Mais pour comprendre la rupture de 1793, il faut tâcher d'envisager comment Mathurin a reçu et interprété ce qui se déroule à partir de 1789. Car si l'Exorde tend à montrer la distance qu'a prise son auteur avec la Révolution, il semble qu'il n'en ait pas toujours été ainsi. Il appartient à l'élite bourgeoise nantaise et à ce titre accueille favorablement les événements de 1789. Fin 1788, il est signataire d'une pétition pour la reconnaissance des droits du tiers-état qui est transmise à Louis XVI par onze députés nantais. S'il ne s'engage pas politiquement dans sa cité, plusieurs de ses amis et membres de sa famille occupent des postes dans l'administration communale ou publient des feuilles engagées à l'exemple de son neveu Antoine $\operatorname{Peccot}^{22}$. Ainsi, Mathurin suit de très près la gestion de la ville, dès la fin 1788 où un nouveau conseil se met en place avec son beau-père Julien Leroux, François Mellinet et le docteur Laënnec, deux de ses plus proches amis. Ingénieur de la garde nationale depuis

(21) Cette base de données, résultat du travail dirigé par François-Joseph Ruggiu et JeanPierre Bardet (CNRS/GDR n²649 et Archives nationales), est accessible à l'adresse suivante : www.ecritsduforprive.fr/accueilbase.htm.

(22) Antoine Peccot est le fondateur, en 1790, de la Chronique de la Loire-inférieure dont le ton est très provoquant, particulièrement à l'égard du clergé. 
octobre 1790, Mathurin est chargé des défenses de la ville, notamment contre les assauts vendéens. Quand, le 13 décembre 1792, une nouvelle municipalité s'installe avec René Gaston Baco, Antoine Peccot et Antoine Crucy deviennent officiers municipaux. Le 2 janvier 1793, il paraphe une délibération du conseil de la commune dénonçant les agissements parisiens et appelant à plus de modération ; au printemps 1793, il signe de nouveau une pétition, celle des gardes nationaux, qui appelle les administrateurs à plus de fermeté dans la gestion des denrées du département de LoireInférieure $^{23}$. Mais son engagement en faveur de la Révolution se perçoit surtout au travers de ses activités d'architecte et d'artiste lorsqu'il est chargé de l'édification de la colonne Louis XVI et qu'il invite le peintre Jacques-Louis David, son ancien condisciple à Rome, pour la réalisation d'une immense toile allégorique représentant l'engagement de Nantes dans la défense de la Révolution. L'histoire et les péripéties de la construction de la colonne Louis XVI sont directement liées à l'histoire politique de la Révolution et intimement mêlées aux prises de position de Mathurin. L'origine de cette colonne s'inscrit dans les événements de la fin 1788, à la suite de la prise de position de Mathurin à l'occasion de la pétition sur les droits du Tiers portée à Versailles. L'idée est d'ériger un «monument de reconnaissance ${ }^{24}$ » à l'égard de Louis XVI, une idée qui subit des transformations au regard des événements politiques de 1789-1790. Si une statue en pied de Louis XVI doit toujours être placée au sommet du monument, celui-ci est désormais dédié à la Liberté à la suite d'un projet cosigné par quatorze architectes nantais le 15 janvier $1790^{25}$. Mathurin, qui coordonne l'ensemble, en expose les objectifs : «J'avais pris pour programme le Serment décrété par l'Assemblée Nationale, la Nation, la Loi, et le Roi. Le piédestal représentait la Nation par les faisceaux réunis et liés ensemble, portant la colonne, pouvoir législatif, avec les noms des députés en aspirail [ ?]. Au-dessus de la colonne est un piédestal pour recevoir Louis XVI représentant le pouvoir exécutif $»^{26}$. Le chantier mit beaucoup de temps à démarrer et il fallut attendre le 24 juin 1790 pour que la première pierre soit posée. Le sculpteur Lamarie, chargé de fondre l'effigie en cuivre, prit du retard et la fuite de Varennes modifia son travail. La Société des Amis de la Constitution obtint l'autorisation de la municipalité

(23) AD Loire-Atlantique, L/598 (1793).

(24) Claude COSNEAU, op. cit., p. 63. cit., p. 64

(25) AM Nantes, D1 registre $n^{\circ} 2$ f $^{\circ} 68-69$ (15 janvier 1790), cité par Claude CoSNEAU, op.

(26) AD Loire-Atlantique, 121 J 5, fonds Crucy, Mathurin Crucy, architecte (1791). 
d'ériger une figure de la liberté en lieu et place de celle du souverain. Mathurin qui dirigeait toujours l'édification du monument proposa une ornementation faite de dédicaces «à la municipalité, au département et au district, aux architectes, à la nation française, aux citoyens de Nantes qui ont présenté la première requête au roi, aux jeunes citoyens de Nantes qui sont partis pour aller secourir leurs frères de Rennes, à l'Assemblée Nationale, aux vainqueurs de la Bastille, à toutes les gardes nationales de France, à toutes les Sociétés des Amis de la Constitution de France et d'Angleterre $\gg^{27}$.

Les années 1790 et 1791 sont riches en projets monumentaux à la gloire de l'engagement de Nantes ou de ses militaires dans la Révolution dont Mathurin est l'un des inspirateurs ou concepteurs. Le projet le plus important est celui qui le pousse à demander à son ami Jacques-Louis David de représenter le maire de Nantes, Kervégan, dans un tableau allégorique représentant l'engagement de la municipalité dans la Révolution. Malgré la venue de David au printemps 1790, le tableau ne fut jamais réalisé. Toutefois, il signifie à nouveau l'implication de Mathurin dans la Révolution et sa volonté de l'inscrire dans les arts et les monuments de la ville. Tranchant avec les lignes de l'Exorde, l'enthousiasme de l'architecte est réel entre 1789 et 1791 . Homme modéré, ces premières années lui semblent être riches de promesses. La déclaration de guerre de 1792, les premiers heurts entre girondins et montagnards et la perception d'une certaine radicalité parisienne depuis Nantes ont refroidi les espoirs de Mathurin qui prend ses distances avec la vie politique à partir de la Terreur.

\section{La rupture Carrier}

Plus que la Terreur proprement dite sur laquelle Mathurin Crucy n'apporte aucun jugement direct, l'épisode Carrier polarise en revanche le propos de l'architecte. Les agissements de ce représentant en mission ont déjà retenu l'attention de nombreux historiens ${ }^{28}$. L'homme qui « devient brutal quand il cesse d'être silencieux ${ }^{29} »$ arrive à Nantes le 8 octobre 1793 dans un climat tendu car, si la ville a réussi à repousser l'assaut

(27) Ibidem.

(28) Jean-Clément MARTIN, La Loire-Atlantique dans la tourmente révolutionnaire (17891799), Nantes, Reflets du Passé, 1989 ; Corinne GoMEZ-LE CHEVANTON, Carrier et la Révolution française, La Crèche, Geste éditions, 2004 ; Jean-Joël BRÉGEON, Carrier et la terreur nantaise, Paris, Perrin, 2002 : Pour une synthèse sur le climat nantais : Samuel GUICHETEAU, « La Terreur sociale à Nantes (été 1793-hiver de l'an II) », dans Michel BIARD (dir.), Les politiques de la terreur (1793-1794), Rennes, PUR, 2008, p. 307-319.

(29) Gaston MARTIN, Carrier et sa mission à Nantes, Paris, PUF, 1924, p. 42. 
des Vendéens fin juin 1793, le nouveau gouvernement parisien perçoit des velléités fédéralistes. Il est difficile de connaître le positionnement de Mathurin mais il est destitué de sa charge d'architecte-voyer par JeanBaptiste Carrier le 17 novembre 1793. À cette époque plusieurs membres de la famille et des amis de Mathurin sont dans la tourmente, particulièrement après la mort du général Léchelle. La rumeur transforme ce mystérieux décès en assassinat et Carrier trouve là un prétexte pour lancer plusieurs arrestations à Nantes et dans le département. Le beau-père de Mathurin, Julien Leroux, son neveu Antoine Peccot, et son associé dans une affaire de café, Jean-François Duparc, sont arrêtés. Finalement, une colonne de 132 prisonniers est envoyée à pied à Paris le 27 novembre. Pourquoi Crucy échappe-t-il à cette arrestation collective ? De nombreuses attestations de son engagement dans la Révolution parviennent à Carrier à l'instar de celle des gardes nationaux qui entendent rappeler que « Nous, officiers d'État Major de la garde nationale certifions que le citoyen Crucy, ingénieur de la garde nationale depuis son organisation provisoire au mois d'octobre 1790, en a rempli les fonctions avec zèle, dans toutes les fêtes publiques, et que, depuis la guerre contre les brigands de la Vendée, il a consacré son temps et ses talents aux ouvrages nécessaires à la défense de la ville, avec le dévouement d'un bon républicain $»^{30}$. Mais Crucy n'est pas le seul à pouvoir bénéficier d'un brevet de bon révolutionnaire. C'est par exemple le cas de Julien Leroux qui, malgré de nombreux documents témoignant de sa probité républicaine, est suspecté de conserver une partie des cuirs et peaux dans sa tannerie et de les écouler au prix fort. Mathurin n'est donc pas inquiété mais il quitte la ville à la fin novembre 1793 et son propos à ce sujet est relativement ambigu : «Carrier vint à Nantes. Je fus proscrit et destitué de ma place d'architecte de la ville de Nantes ; obligé de sortir de la ville, je me retirai dans les départements de l'intérieur de la France où il y a des forêts $»^{31}$. « Proscrits », « obligés de sortir de la ville », voilà une interprétation bien personnelle. Carrier s'est contenté de le remplacer à son poste d'architecte, rien de plus. Toutefois, une peur légitime s'est emparée de plusieurs personnalités nantaises et certaines, à l'image de Mathurin Crucy, ont fait le choix de partir en attendant des jours meilleurs. Car l'étau semblait se resserrer sur la famille : à côté de la destitution de l'aîné, les chantiers de la Piperie et de Basse Indre, destinés à approvisionner la République en navires, avaient été réquisitionnés, dépouillant les trois 
frères de leur outil de travail et la source de leur revenu. Dans ce contexte, le départ de Mathurin s'explique, même si la dramaturgie qu'il instille dans l'Exorde est très exagérée. Le même parti pris teinte les lignes qu'il consacre à son retour à Nantes : « Après le départ de Carrier, la commune de Nantes me rappela à ma place, la délibération était honorable et flatteuse, il n'était guère possible de me refuser aux vœux de mes concitoyens, malgré la répugnance que j'avais à venir habiter avec des hommes méchants et jaloux $\gg^{32}$. La délibération du conseil municipal est plus que flatteuse ; elle succède très rapidement au départ de Carrier, convoqué à Paris le 16 février 1794 tandis que Mathurin est rappelé le $17^{33}$. Contrairement à ce que laissent envisager les lignes de l'Exorde, Crucy avait de nombreux partisans au sein de la commune. Mais ce passage consacré aux années révolutionnaires révèle en réalité une importante rupture pour Mathurin, la seconde depuis la mort de son frère aîné. L'auteur ne porte aucun regard politique sur la période qu'il traverse et le détail qu'il dresse de l'activité de Carrier n'existe que pour mettre en exergue son renvoi. Comme lors du décès de JeanGilbert, l'écriture se veut empreinte de plus de gravité. En 1783, Mathurin aurait pu abandonner sa charge pour diriger les affaires familiales et le récit qu'il fait de ce moment laisse entrevoir l'horreur de cette perspective pour l'auteur. En 1793, Carrier ne le lui laisse guère le choix, provoquant chez l'architecte du ressentiment, voire de la colère. Au delà de sa destitution, c'est à son identité que l'on s'attaque. Une fois de plus, Mathurin s'érige en victime et fulmine contre le sort qui lui est réservé. Mais cette dénonciation ne dépasse jamais son cas personnel pour déplorer les traitements réservés à sa famille ou à ses proches, loin d'être épargnés pendant ces quelques mois. Ce parti pris de rédiger une autobiographie très égocentrée peut s'expliquer par les raisons qui ont poussé le scripteur à produire ce texte. L'hypothèse formulée en introduction invite à penser l'Exorde comme un document permettant de fournir des éléments à l'avocat de Mathurin, chargé de le défendre contre ses frères en 1813. Un tel contexte peut sans doute expliquer à la fois l'exagération manifeste de la posture de victime mais également la disparition de tous les acteurs qui ne sont pas immédiatement impliqués dans les difficultés de Mathurin. Toutefois, si ces éléments sont à prendre en compte, l'Exorde dévoile aussi l'égocentrisme de son auteur. Au-delà, c'est bien la Révolution en tant qu'événement qui disparaît de l'Exorde. Si certains écrits du for privé permettent d'appréhender de grandes périodes 
de ruptures politique, économique ou sociale de manière originale ${ }^{34}$, rien de tel avec l'écrit de Mathurin.

\section{La liquidation de la société L. Crucy frères}

\section{Le poids des dissensions familiales}

Des trois grandes thématiques que Mathurin aborde dans son autobiographie, les dissensions familiales occupent une place majeure, de loin la première dans la mesure où l'évocation de sa jeunesse et des bribes de sa vie professionnelle ne sont présentes que pour mettre en exergue la violence et l'injustice de sa condition en 1813. Depuis 1809, Mathurin aspire à une retraite qu'il considère méritée ${ }^{35}$. Mais la mésentente qui l'oppose à ses deux frères retarde considérablement la liquidation des affaires Crucy. Dans l'Exorde, Mathurin tente, à force de chiffres, de démontrer qu'il a été spolié. Il dépeint alors des portraits peu flatteurs de ses frères Louis et Antoine en se posant d'emblée comme la victime de leurs malversations. Sans entrer dans le détail des sommes dues et des successions, il est important de revenir sur le parcours singulier de ces trois hommes perçus de prime abord comme très liés. Rappelons que de cette fratrie dont Mathurin dresse les contours, les deux sœurs, Anne et Michelle, sont absentes. Mathurin n'évoque jamais ces deux femmes pourtant au cœur d'une fêlure familiale passée sous silence. Car si Mathurin fait remonter ses difficultés à la brouille qui survient avec son frère Louis en 1805, la famille se déchire depuis 1788 à cause du règlement de la succession de leur père, décédé en 1785. À la veille de la Révolution, Mathurin, Louis et Antoine rompent leurs liens avec leurs sœurs en quittant le quartier Saint-Léonard, le lieu de leur naissance et de leur jeunesse, pour le quai de la Fosse où les négociants et les armateurs s'installaient. Au début de l'Empire, la société L. Crucy frères est au faîte de sa puissance, monopolisant les marchés de construction pour l'État et engageant de nombreux chantiers pour la marine de commerce et les armateurs en course. Malgré les aléas politiques et la crise économique qui caractérisent le Directoire, les trois frères ont bâti de solides fortunes et ont fait croître leur patrimoine, comme en témoignent de

(34) François-Joseph RugGIU, « Un événement de la Fronde ? La mort de Charles $\mathrm{I}^{\mathrm{er}}$ d'Angleterre dans les écrits de for privé français », dans Jean-Pierre BARDET, Élisabeth ARNOUL, François-Joseph RugGIU (dir.), Les écrits du for privé en Europe du Moyen-Âge à l'époque contemporaine. Enquêtes, Analyses, Publications, Bordeaux, Presses Universitaires de Bordeaux, 2010, p. 535-554.

(35) AD Loire-Atlantique $121 \mathrm{~J} 22-23$ folio 8, fonds Crucy, Exorde (1813). 
nombreux documents ${ }^{36}$. Sous cette apparente prospérité, l'Exorde donne à voir l'incapacité de Mathurin et de ses frères à gérer leurs affaires et l'organisation singulière qui caractérise cette entreprise de construction navale. Géographiquement éclatée sur plusieurs sites - Nantes, Basse-Indre, Couëron, Paimboeuf, Lorient et Rochefort - cette société possède son siège social au quai de la Fosse mais dépend de Paris pour ses marchés et son financement. Mathurin évoque une relation directe avec le ministre et se plaint de la nécessité répétée de se rendre à Paris pour négocier les contrats avec Denis Decrès tout au long de l'Empire. Mais ces voyages parisiens lui permettent surtout d'obtenir des avances de la banque Récamier qui est partie prenante de l'entreprise à partir de 1801. Dans cette organisation, la distribution des rôles entre les trois frères est très floue d'autant que Mathurin minimise son engagement dans la gestion proprement dite : « [...] les affaires allaient avec activité, beaucoup de zèle et d'ordre. Ma confiance en mes frères était si parfaite que je n'eus jamais aucun soupçon ni aucune crainte. Je n'ai point fait vérifier les livres ni la caisse, tout était en commun et sur la bonne foi : les biens que nous avions acquis, les marchandises qui se renouvelaient chaque jour appartenaient aux trois frères. Je n'ai donc pas eu à me plaindre tant que cette union a existé $»^{37}$. En fait, l'analyse des pièces comptables et de la correspondance de l'entreprise montre un plus grand investissement de l'aîné, particulièrement après 1800 où il apparait très fréquemment comme la courroie de transmission entre Paris et les chantiers. Louis eut davantage un rôle de contremaître en suivant le déroulement des constructions tandis qu'Antoine se chargeait de la gestion des fournisseurs et du matériel. Toutefois, rien d'immuable dans cette organisation. Au reste, ce qui ressort à la lecture de l'Exorde est l'impréparation de ces trois hommes. Leur banquier Récamier ne semble pas davantage prédisposé à répondre au gigantisme propre à la construction navale militaire. Dès 1802, il s'inquiète des sommes qu'il doit avancer aux Crucy : « l'aperçu que vous me donnez des travaux entrepris et dont l'évaluation s'élève à 6.680 .000 francs dépasse de beaucoup l'idée que je

(36) L'inventaire après décès d'Antoine Crucy, mort en 1815, montre que le cadet de la fratrie possédait encore un patrimoine important notamment un appartement à Nantes, une propriété à la campagne et de nombreux terrains même si l'ensemble fut grevé par de nombreuses dettes (AD Loire-Atlantique A $121 \mathrm{~J}$ 1, fonds Crucy, Famille et patrimoine, inventaire après décès d'Antoine Crucy (19 avril 1815)). Mathurin semble mieux loti. Il est également plus prudent puisqu'il procède à un partage anticipé de ses biens entre ses trois enfants au moment où son fils aîné se marie (AD Loire-Atlantique $121 \mathrm{~J}$ 1, fonds Crucy, Famille et patrimoine, partage anticipé des biens de Mathurin Crucy (4 décembre 1815)).

(37) AD Loire-Atlantique 121 J 22-23 folio 9, fonds Crucy, Exorde (1813). 
m'en était faite $\gg^{38}$. Quatre ans plus tard Récamier fait faillite. Il est difficile de préciser la responsabilité des Crucy dans cet accident économique mais ce qu'ils devaient représentait un peu plus de la moitié des dettes de la banque ${ }^{39}$.

La faillite Récamier entraine celle de l'entreprise Crucy et solde l'entente entre les trois frères. La nécessité d'apurer les comptes fait émerger l'amateurisme qui a été la règle depuis le début et Mathurin en fait porter la responsabilité à Louis : « Mon parti pris je demande des comptes et un état de situation, je connaissais les obligations que nous avions prises avec le gouvernement, il allait de notre devoir de les remplir. J'étais décidé à ne mettre ma signature nulle part que l'on ne me fit connaître l'état de nos affaires, pour savoir ce que nous possédions et ce que nous devions. Louis nous l'avait promis dix fois et jamais il n'a tenu sa promesse $»^{40}$. Nul doute que cette mauvaise gestion incombe aux trois frères mais, quelques mois avant la liquidation, l'Exorde rapporte que Louis se serait servi du travail de son frère à Paimboeuf pour établir des chantiers de construction navale pour son propre compte et celui de son fils. Mathurin vécut cet épisode comme une trahison et en ressort profondément blessé, bien décidé à ne plus accorder sa confiance à Louis. Dans ce contexte, et faute d'une comptabilité précise de l'établissement, la liquidation de celui-ci traîne de nombreuses années pour s'achever partiellement en 1813, ce qui n'empêche pas Louis et son fils de répondre à des commandes de l'État depuis Paimboeuf et à Mathurin et Antoine de faire de même aux chantiers de la Piperie, à Basse Indre et Coüeron. La destinée de ces deux entreprises concurrentes n'est pas plus heureuse, contraintes de composer avec un trésor impérial exsangue, la pénurie de matériaux de construction et l'ensablement de la Loire, ce dernier souci handicapant plus particulièrement Mathurin et Antoine. Dès 1809 , le sort des chantiers semble jeté, d'autant que Mathurin fait part à son frère du désir de se retirer des affaires. Cette séparation provoque de nouvelles tensions dans la mesure où Antoine ne peut dédommager immédiatement Mathurin pour son départ de l'entreprise malgré un accord signé le 9 juillet $1809^{41}$. Par ailleurs, Louis conteste ce partage et estime avoir été lésé, alors que la liquidation de 1806 n'est pas encore terminée. La fin de l'Exorde est une accumulation de chiffres auxquels il est bien difficile d'apporter crédit. En 1813, Mathurin, las et épuisé de tant de

(38) Yves Cossé, op. cit., p. 140.

(39) Ibidem.

(40) AD Loire-Atlantique $121 \mathrm{~J}$ 22-23 folio 9, fonds Crucy, Exorde (1813).

(41) Ibidem, folio 12. 
conflits, semble accepter le sort qui lui est réservé par les accords arrachés aux différentes parties. L'aventure industrielle des chantiers navals a eu raison des liens qui unissaient les trois frères : «Je ne parlerai pas des procédés d'Antoine à mon égard ils sont affreux ; j'avais été trahi dans l'amitié de Louis, je l'ai été par celle d'Antoine, les chicanes qu'il me fait dans mes jouissances annoncent un homme qui n'est pas délicat. Je ne suis pas plus heureux avec l'un qu'avec l'autre, voilà où l'amitié et la confiance m'ont conduit $»^{42}$.

\section{Le poids de l'État}

En arrière-plan de l'autobiographie de Mathurin, on mesure le poids de l'État, voire sa violence, durant la Révolution et l'Empire. Cette violence économique préexiste aux régimes politiques qui se succèdent à partir de 1789, la monarchie n'ayant pas épargné ses partenaires dans ce domaine, même si l'administration de la Marine mettait tout en œuvre pour sauver ses partenaires les plus stratégiques. Les frères Crucy appartiennent à une catégorie d'acteurs indispensables pour soutenir l'effort de guerre mais ils sont maltraités, pressurés et tributaires de la bonne santé financière de leur commanditaire. Tous les fournisseurs, munitionnaires ou entrepreneurs au service de l'économie militaire n'ont pas accumulé de confortables fortunes, loin s'en faut. Pourtant, sans aller au devant d'un travail comptable complexe qui reste à faire, les gains générés par les chantiers Crucy pouvaient assurer à leur propriétaire une respectable retraite. Même s'il s'agit de lire les indications de Mathurin avec recul, les sommes débattues entre les frères à partir de 1806 apparaissent ridiculement faibles au regard de l'activité réalisée depuis le démarrage des chantiers en 1793. Entre cette date et 1806 , le chiffre d'affaires de la société L. Crucy frères s'élèverait à près de 25 millions de francs. Sans présumer du bénéfice net réalisé, les Crucy auraient dû être capables de faire face à la faillite Récamier, même dans le contexte d'une gestion peu rigoureuse. Or l'État est incapable de payer ses traites dans les temps. La Marine fait construire à découvert des navires depuis 1801 obligeant les Crucy à demander des avances toujours plus importantes à leur banque. À cette date, les dettes du gouvernement s'élèvent à 284000 francs contre près d'un million en 1808. Etienne Poussielgue, soutien financier des Crucy, avait mis en garde Mathurin dès 1802 : «Le gouvernement ne donne de l'argent que quand il ne peut obtenir les objets dont il a absolument besoin. Il faut que vous cessiez tous

(42) Ibid., folio 13. 
vos travaux à l'instant même où, quand vous avez rempli fidèlement vos engagements envers le gouvernement, il suspend l'exécution de ceux qu'il a contractés avec vous. Il faut prendre ce parti sans hésiter et le prendre chaque fois que le cas arrivera, sans vous laisser gagner par des promesses. On vous en fera tant que vous voudrez mais on ne les tiendra pas sans une nécessité absolue et cette nécessité ne sera jamais sentie qu'autant que les travaux seront suspendus $»^{43}$. En creux, ce courrier montre l'expectative dans laquelle devait être plongé Mathurin. Mais les frères Crucy avaient-ils la possibilité d'interrompre les travaux ? Rien n'est moins sûr, d'autant que l'amiral Decrès se montre très retors à l'égard des entrepreneurs pour qu'ils avancent plus rapidement les unités en chantier, particulièrement après 1808 où le ton des courriers du ministre devient de plus en plus radical ${ }^{44}$. Les dernières années de l'Empire sont très difficiles pour les chantiers navals. Les matières premières sont de plus en plus rares et atteignent des prix prohibitifs. Le ministère refuse fréquemment de prendre en compte ces éléments pour renégocier les contrats de sous-traitance avec ses partenaires. De pétitions en mémoires argumentatifs, les Crucy ne parviennent que très rarement à obtenir gain de cause. La violence de l'État s'exprime au travers de cette relation brutale plaçant les entrepreneurs en perpétuelle position de faiblesse, les obligeant à supplier le Pouvoir pour ne pas mourir. Par ailleurs, le rôle joué par l'amiral Decrès dans la séparation des frères Crucy est troublant. Dans l'Exorde, Mathurin évoque le départ en septembre 1805 du fils de Louis pour un mystérieux voyage à Paris :

« [...] J'appris que Louis le fils était parti pour Paris sans rien dire à personne. Ce départ me surprit, $\mathrm{j}$ 'en fis des reproches au père qui me dit qu'il avait profité de l'occasion d'un ami pour faire ce voyage [...] je le crus. Un mois ou deux après, Antoine vint me trouver à la campagne pour m'annoncer que Louis le fils avait entrepris une frégate et une corvette pour construire à Paimboeuf, je ne puis le croire. [...] J'engage Antoine d'aller voir Louis et d'avoir une explication amicale avec lui pour connaître la vérité du fait. Mes deux frères se quittent très mécontents l'un de l'autre. Louis vint à la campagne se plaindre de la conduite d'Antoine et des propos injurieux qu'il lui avait tenu, je lui demandais si véritablement son fils avait passé un marché avec le ministre pour construire à Paimboeuf, il me dit

(43) Ibid., 21 J 38, correspondance, Éienne Poussielgue à Louis Crucy (1802).

(44) Ibid., $121 \mathrm{~J}$ 41, correspondance, l'Amiral Decrès à Louis Crucy (1812). 
oui. [...] Je lui ai parlé de l'inconvenance de cette conduite et du désordre qui allait en résulter dans notre famille et dans nos affaires $»^{45}$.

Pourquoi encourager la division des chantiers alors que la société L. Crucy frères peine déjà à remplir ses obligations ? À la décharge du ministre, la seule parole de Louis Crucy ne suffit pas à le convaincre puisqu'il entreprend d'en savoir davantage auprès du commissaire de la marine à Lorient :

« La maison Crucy s'est montrée jusqu'à présent digne de la confiance du gouvernement par le zèle qu'elle a développé dans les entreprises qui lui ont été confiées et d'après cette considération je serais disposé à accueillir les propositions qui me sont faites par Monsieur Louis Crucy fils parce que j'espère que le jeune homme suivra l'exemple qu'il a reçu de ses parents. D'ailleurs, la construction d'un nouveau chantier à Paimboeuf me paraitrait devoir être ultérieurement utile à la marine militaire ainsi qu'au commerce maritime. Cependant, avant de donner suite à ces propositions, je désire connaître votre opinion sur le nouvel établissement. [...] J'attends de vous à cet égard la plus grande impartialité ${ }^{46}$.

Toutefois, dans les échanges ultérieurs, il n'est jamais question des conséquences d'une telle création sur l'équilibre fragile de la société existante ; dès 1806, les frégates Clorinde et Rivoly sont en chantier à Paimboeuf. Après la liquidation de la société L. Crucy frères, Mathurin et Antoine décident de poursuivre les constructions au profit de l'État et l'aîné se rend à Basse-Indre pour diriger personnellement les travaux : «[...] je me charge des constructions de trois frégates qui doivent être au chantier de la Basse-Indre, j'en avais contracté l'engagement avec le ministre, j'étais jaloux d'en remplir l'obligation. Je laissai Antoine à Nantes suivre ses opérations. Je partis pour la Basse Indre, où je restai près de trois ans $»^{47}$. Dès cette époque, et jusqu'en 1814, deux entreprises de construction navale se partagent l'estuaire de la Loire et sous-traitent des unités pour le compte de l'État. Loin de simplifier les opérations, cette concurrence a des conséquences catastrophiques pour la gestion des approvisionnements et le partage du matériel qui était, jusqu'en 1806, commun aux trois frères. Passée l'heureuse période 1806-1808 où les deux chantiers parviennent à lancer les navires commandés, la fin de la période voit l'accumulation des

(45) Ibid., 121 J 22-23 folio 9, fonds Crucy, Exorde (1813).

(46) Cité par Yves CossÉ, op. cit., p. 177.

(47) AD Loire-Atlantique 121 J 22-23 folio 10, fonds Crucy, Exorde (1813). 
difficultés et aucune des deux infrastructures ne parvient à surmonter ces épreuves. À bout de force et de crédit, Antoine, qui a poursuivi l'activité des chantiers malgré le départ de Mathurin en 1809, meurt en 1815 et son entreprise ne lui survit pas. Juridiquement, Louis et son fils cèdent les ateliers de Paimboeuf fin 1813 mais s'engagent dans un bras de fer avec l'État pour obtenir des indemnités en réparation des impayés accumulés.

Les écrits du for privé peuvent être étudiés de bien des manières et l'Exorde illustre cette variété d'approche. De prime abord, cette autobiographie permet de mettre en avant le processus de construction de l'identité individuelle de Mathurin Crucy et de comprendre comment celui-ci s'est perçu. Faisant corps avec son métier, se confondant pour ainsi dire avec lui, Mathurin interprète tous les obstacles à l'exercice de l'architecture comme une violence insupportable. Les événements, intimes - la mort de son frère aîné - ou de portée plus générale et collective - la Révolution française - ne sont vécus qu'à l'aune des perturbations induites sur ses possibilités d'exercer son art. Toutefois, la violence ressentie par l'auteur n'est pas uniquement le résultat d'une perception personnelle. Les rapports entretenus avec le ministère de la Marine ont entraîné une violence objective déstabilisant Mathurin et participant à l'éclatement de la fratrie. Mais à bien des égards, l'Exorde présente également un intérêt évident pour saisir la vie de la société L. Crucy frères sous un angle différent de celui communément perceptible par les archives comptables, les marchés ou les procès verbaux de visite des constructions. Face à la pauvreté des fonds entrepreneuriaux pour l'Ancien Régime, la Révolution et l'Empire, l'usage des documents du for privé se révèle très précieux. L'Exorde renseigne sur les ressorts organisationnels de l'entreprise - gestion, partage des responsabilités, relations avec l'État - et l'enracine dans une histoire familiale complexe, deux aspects d'un abord peu évident. Même s'il s'agit d'avoir un regard distancié, le récit proposé par Mathurin Crucy donne une profondeur de champ inédite pour restituer cette trame entrepreneuriale. Les chantiers de construction disparaissent avec l'Empire et Mathurin termine sa vie en travaillant à la construction de quelques églises dans le département, la Halle aux Toiles de Nantes et la cathédrale de Rennes. Si sa mort, le 7 novembre 1826, ne provoque pas un grand émoi, la nécrologie publiée dans le Journal de Nantes et de la Loire-inférieure quelques jours plus tard consacre finalement la quête identitaire de Mathurin en ne retenant de sa vie que son action d'architecte, oubliant sa participation dans les premiers chantiers de construction navale privés de la Révolution et de l'Empire : «La mort vient d'enlever un des hommes distingués de la ville de Nantes : Monsieur Mathurin Crucy, architecte, correspondant de l'Institut et membre 
de la Société Académique de la Loire-Inférieure, décédé le 7 du courant. Parmi les nombreux travaux qui assurèrent une renommée durable au nom de Mathurin Crucy il suffit de citer les beaux monuments de la Bourse et de la Salle de spectacles ${ }^{48}$.

David PlouvieZ

Université de Nantes

Centre de Recherche en Histoire Internationale et Atlantique (CRHIA), UFR Histoire, Histoire de l'Art et Archéologie, Chemin de la Censive du Tertre, BP - 8122744312 Nantes Cedex 3 